Available online at GSC Online Press Directory

GSC Biological and Pharmaceutical Sciences

e-ISSN: 2581-3250, CODEN (USA): GBPSC2

Journal homepage: https://www.gsconlinepress.com/journals/gscbps

(REVIEW ARTICLE)

\title{
Application of artificial intelligence (AI) in food industry
}

\author{
Chidinma-Mary-Agbai $1{ }^{*}$ \\ Department of Food Science and Technology, Federal University of Technology Owerri, Owerri, Nigeria.
}

Publication history: Received on 29 September 2020; revised on 09 October 2020; accepted on 15 October 2020

Article DOI: https://doi.org/10.30574/gscbps.2020.13.1.0320

\begin{abstract}
Artificial intelligence (AI) is the theory and development of computer systems able to perform tasks normally requiring human intelligence. With teeming competition and increasing demand in the food industry, the industry has begun to embrace AI technologies in a bid to maximize profits and explore new ways to reach and serve the consumers. AI has been successfully deployed for applications such as sorting fresh produce, managing supply chain, food safety compliance monitoring, effective cleaning in place systems, anticipating consumer preference and new product development with greater efficiency and savings on time and resources. However, there are challenges to adoption of AI technologies which include cost, cultural changes, expert skill requirements, transparency issues and one track minds. Despite these challenges, researches are on-going on optimized production process using AI but it is important to note that the benefits of AI application in food industry greatly outweigh its challenges.
\end{abstract}

Keywords: Optimized production, Artificial Intelligence, Machine learning, Robotics

\section{Introduction}

Artificial intelligence traditionally refers to an artificial creation of human-like intelligence that can learn reason, plan, perceive or process natural language [1,2]. It is the theory and development of computer systems able to perform tasks normally requiring human intelligence such as visual perception, speech recognition, decision making and translation between languages [3]. Artificial intelligence is a branch of computer science which mainly deals in creating machines that are developed in such a way that it acts like a human being. John McCarthy (the father of AI) defined AI as "the science and engineering of making intelligent machines especially intelligent computer programs" [4]. Machine learning and deep learning are two of the most commonly used algorithms in the field of AI. These models learn from data and are used by people, companies and government agencies to make predictions. Today, machine learning models are being developed to deal with the complexity and variety of data in the food industry [5].

In food industries, where the design of standard reliable procedures to control the quality of products is a major objective and as with other companies, the search for new ways to reach and serve the customers while keeping the costs low have necessitated the deployment of AI to achieve better customer experience, efficient supply chain management, improve operational efficiency, reduction in material movements and vehicle activity and to achieve the best results in the business [6, 7].

\footnotetext{
* Corresponding author: Chidinma-Mary-Agbai; +2348030652334; nmamerry@gmail.com

Department of Food Science and Technology, Federal University of Technology Owerri, Owerri, Nigeria.
} 


\section{Different fields of artificial intelligence}

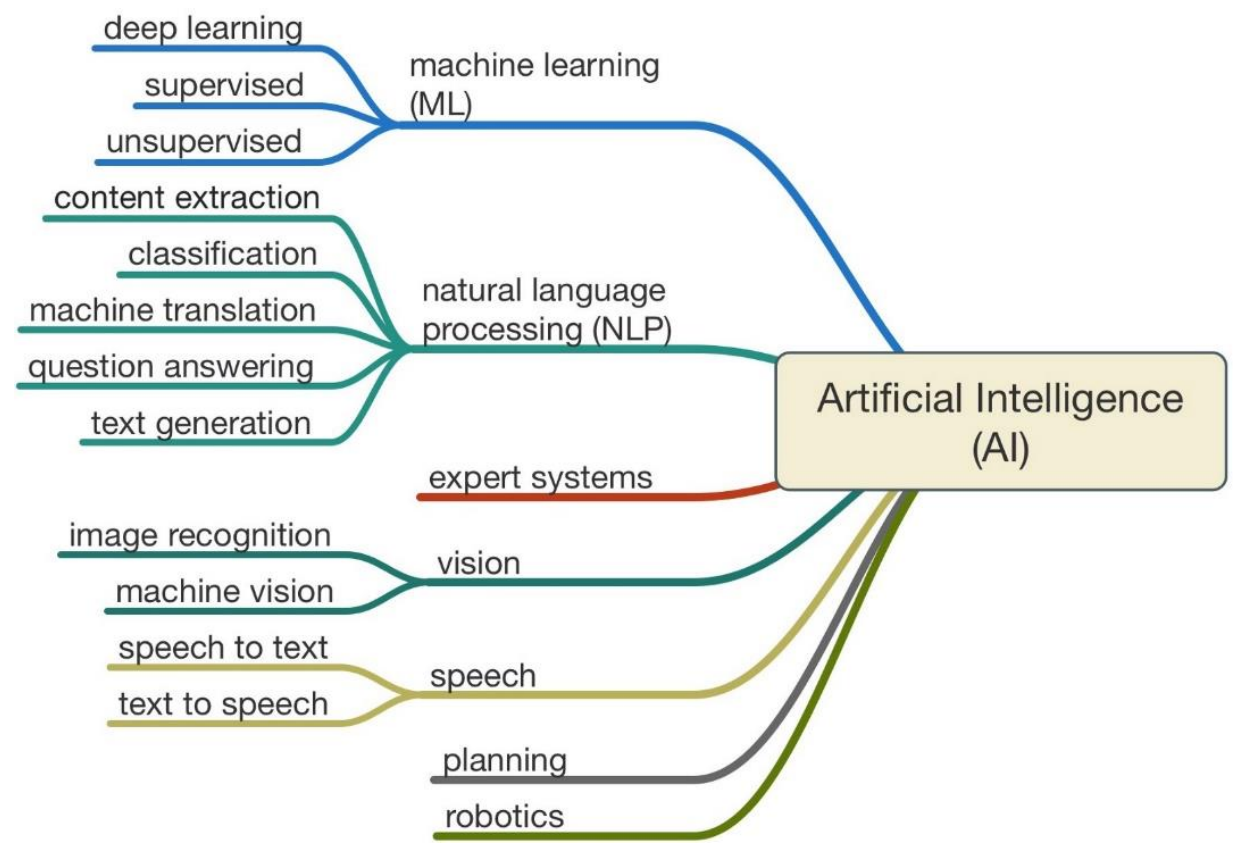

Figure 1 Fields of Artificial intelligence [3]

The most important among them are as follows:

\subsection{Machine Learning (ML)}

It is a scientific study of algorithms and statistical models that computer systems use to effectively perform a specific task without using explicit instructions relying on patterns and inference instead [8]. For example to identify a simple object such as an apple or orange. The target is achieved not by explicitly specifying the details about it and coding it but it is just as we teach a child by showing multiple different pictures of it and therefore allowing the machine to define the steps to identify it like an apple or an orange.

\subsection{Natural Language Processing (NLP)}

Natural Language Processing is broadly defined as the automatic manipulation of natural language, like speech and text, by software. It is a subfield of computer science concerned with the interactions between computer and human languages that is how to program computers to process and analyze large amounts of natural language data [9].

\subsection{Vision}

It is a scientific field which enables the machines to see. Machine vision captures and analyses visual information using a camera, analog-to-digital conversion, and digital signal processing. It seeks to automate tasks that the human visual system can do [10].

\subsection{Robotics}

It is a field of engineering and science that deals with the design, construction, operation and use of robots as well as computer systems for their control, sensory feedback and information processing. These technologies are used to develop machines that can substitute for humans and replicate human actions [11,12]. Robots are often used to perform tasks that are difficult for humans to perform or perform consistently.

\subsection{Autonomous Vehicles}

This is also known as self driving car or robot car. It is a vehicle that is capable of sensing its environment and moving with little or no human input [13]. 


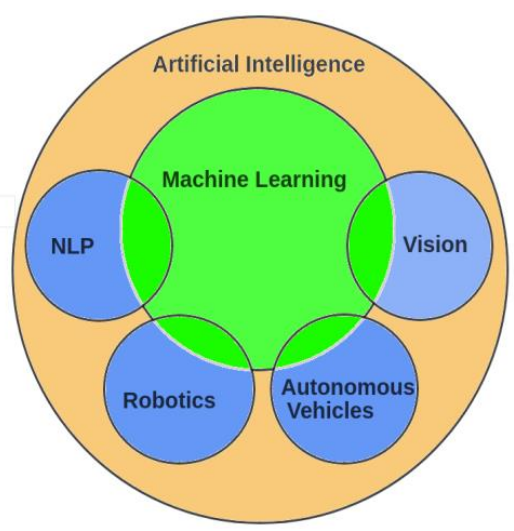

Figure 2 Representation of how machine learning plays a major role in achieving AI [3].

\section{Applications of artificial intelligence in food industry}

\subsection{Sorting Fresh Produce}

One of the biggest challenges faced by food processing plants relates to the irregular availability of feedstock. Food processing plants depend on manual sorting to sift and sort vegetables, leading to loss of efficiency and increased costs [14].

By using Artificial Intelligence, food processing companies can achieve significant automation for food cataloguing, using a combination of cameras, lasers and machine learning to enable food sorting with enhanced efficiency. For instance, by deploying Artificial Intelligence involving sensor-based optical sorting solutions, the tardy time-consuming processes for sorting fresh produce can be removed, leading to higher yield with better quality and lesser wastage [15]. AI is used as a means to better calibrate machines in order to manage several product sizes and reduce waste and costs [1].

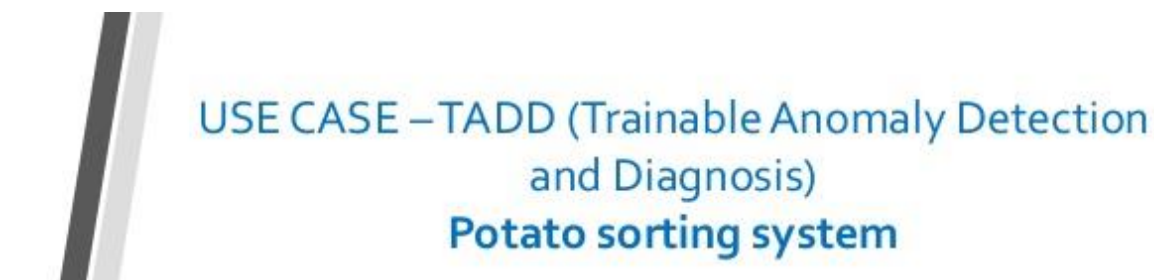

A robotic system that sorts and can detect diseases of potatoes
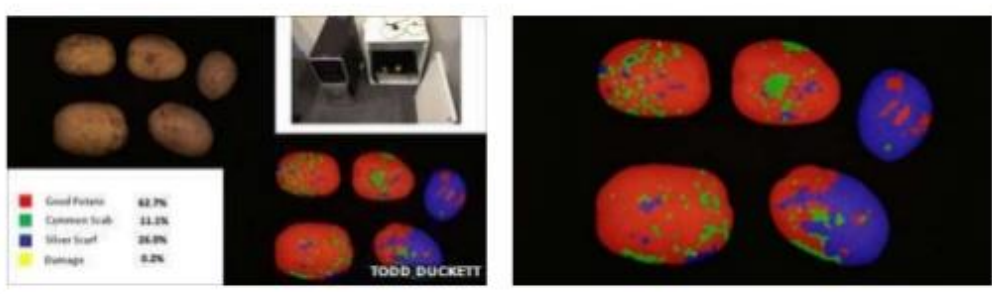

All rights reserved. 2016 http: /l/Al.Business

Figure 3 Potato sorting system [16]. 


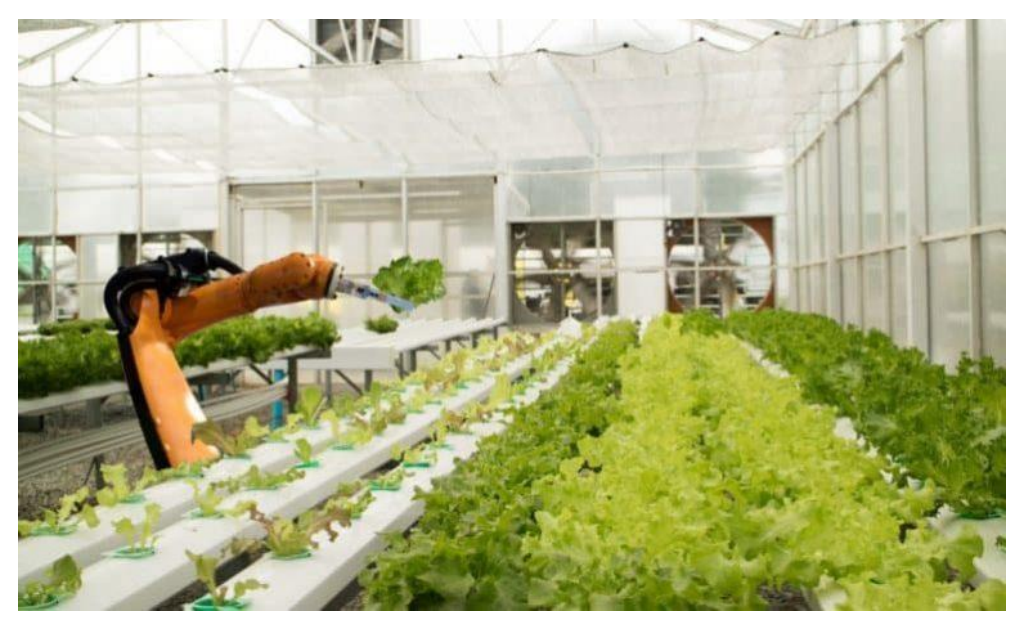

Figure 4 AI enabled sorting machine [17].

\subsection{Efficient Supply Chain Management}

Supply chain management is top priority for all food companies with the increasing need for transparency. The food industry is using AI to improve supply chains through

Food safety monitoring and testing product at every step of the supply chain to ensure compliance to industry and consumer specifications.

More accurate forecasting to manage pricing and inventory [15].

AI-based image recognition technologies enable better and more efficient procurement of produce.

AI also helps in efficient and transparent tracking of produce from farm to the consumer, leading to increased confidence amongst consumers [14].

\subsection{Food Safety Compliance}

AI enabled cameras are used to ensure safety compliance amongst food workers in food facility. This employs facialrecognition and object-recognition software to determine whether workers are complying with good personal hygiene as required by food safety law. If violation is found, it extracts the screen images for review which can be rectified in the real time [5]. The accuracy of this technology is more than 96\% [15].

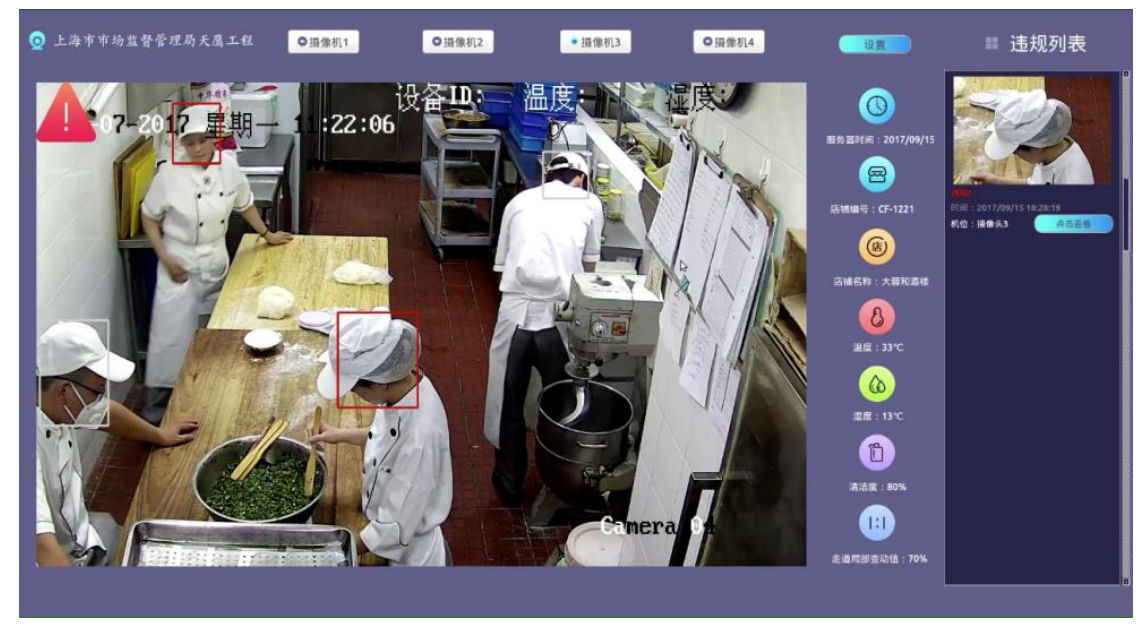

Figure 5 AI enabled camera in a food facility [18]. 


\subsection{Cleaning Food Processing Equipments}

Cleaning in place systems are programmed to clean equipment in timed cycles. This limits human intervention which in turn limits the chances of cross contamination from food borne pathogens but this system operate blindly and are designed for worse case scenarios.

With the help of AI enabled technologies (self-optimizing clean in place system (SOCIP)) which uses ultrasonic sensing and optical fluorescence imaging to measure food residue and microbial debris in a piece of equipment and then optimize the cleaning process [19]. This leads to savings on water, time and energy. The cleaning time is reduced by $50 \%[18]$.

\subsection{Anticipating Consumer Preferences}

Food manufacturers use artificial intelligence-based solutions that enable them to clearly anticipate and model the flavour preferences of their target consumers, and predicting their response to such new tastes. Such Artificial Intelligence-based predictive analytics will help food manufacturers in developing new food products that are closely aligned with consumer tastes and preferences. The Kellogg Company launched AI-enabled technology in 2017 which helps the customer to decide with granola should be chosen from 50 ingredients for making a particular customized product [18]. The AI makes suggestions about what ingredients to add to your granola and lets you know if your ingredients are likely to taste good together or not.

The AI doesn't just help individuals make customized small batches of granola. The general data from flavour combinations, information about what selections people actually make, and what combinations people re-order create a feedback loop refining what flavours people actually like. This source of data will likely provide the parent company very useful information when it comes to deciding what new products to introduce in its much larger brands.

\subsection{Developing New Products}

AI technology uses machine learning and predictive algorithms to model consumer flavour preferences and predict how well they will respond to new tastes. The data can be segmented into demographic groups to help companies develop new products that match the preferences of their target audience [15]. With these, manufacturers could know what products will thrive before the hit the shelves.

Across numerous restaurants and other locations, Coca-Cola has installed self-service soft drink fountains which allow individuals to customize their own drinks. Customers can use these self-service machines to theoretically create hundreds of different drinks by adding different flavours to their base beverages [18]. These thousands of freestyle drink fountains, each dispensing hundreds of different drinks a day, created a massive amount of consumer preference data that Coca-Cola is using AI to analyze. The first product to come from this data was CHERRY SPRITE. Their AI determined that people on their own created a significant amount of cherry-flavoured Sprite and that it would do well as its own standalone product.

\subsection{Front-End or Consumer Facing Applications of Artificial Intelligence}

\subsubsection{Recommedation engines:}

Artificial Intelligence-based food discovery and recommendation engines can help consumers make informed choices on what to eat and what not to eat through applications that learn about consumer's food preferences and requirements [20].

\subsubsection{Apps and Chatbots}

By leveraging Artificial Intelligence-based Virtual Assistants, food restaurants can ensure that customers do not need to wait endlessly before making inquires and process and customize orders. The process is simplified, and contributes to better customer experience [14].

\subsubsection{Self-Ordering AI-based Kiosks}

Artificial Intelligence-based self-ordering machines can contribute to enhanced customer experience by reducing their waiting time, and the need to stand in line for making payment. Such machines can take consumer order, and enable them to make payments directly without human assistance through integrated card readers [21]. 


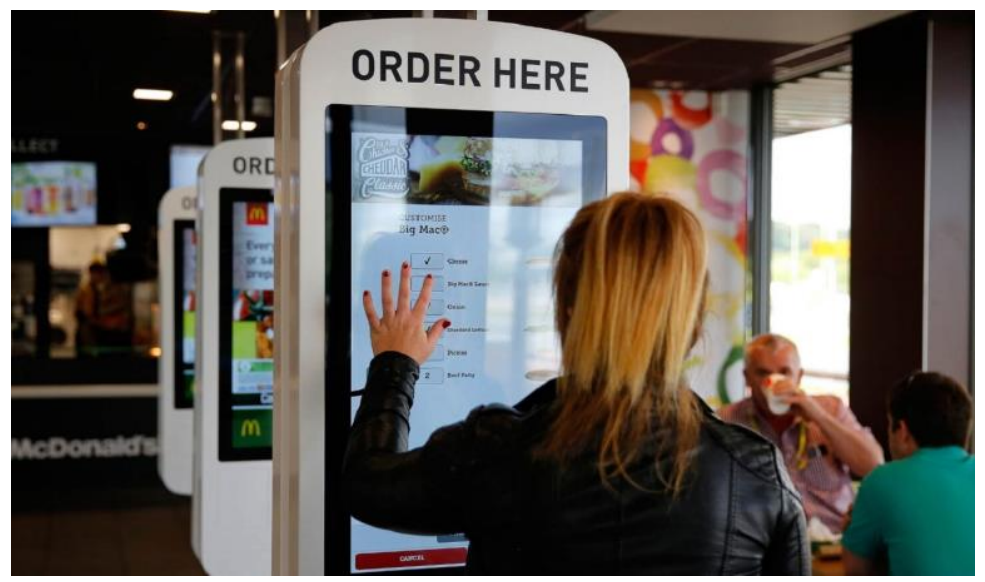

Figure 6 McDonald's Self Ordering Kiosk [21]

\subsubsection{Robots}

In restaurants, robots are making an appearance and enhancing the speed and capacity of food preparation as well as shortening the time for food delivery [14].

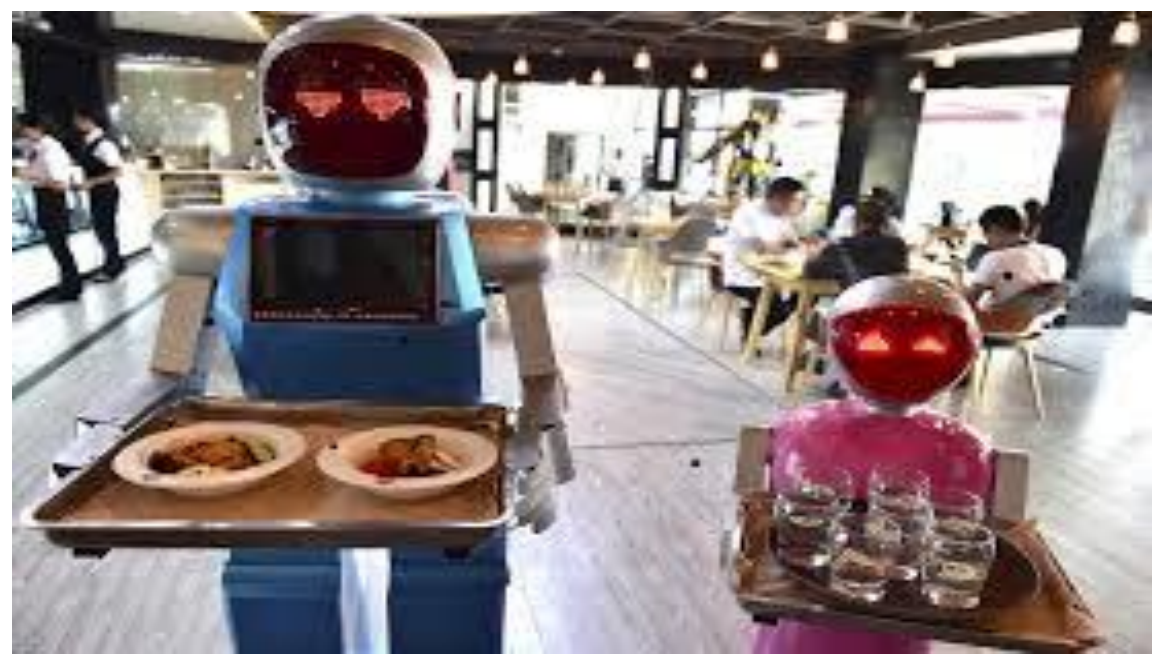

Figure 7 Serving robots [22]. "Hello, I'm your server Xiaolan (left) and this is my assistant Xiaotao. Can we get you a drink?" The restaurant, where these two food-delivering robots work, is real and located in Zhejiang province, China [22]

\section{Challenges to the adoption of artificial intelligence technology in the food industry}

Setbacks to the adoption of AI technology include

The cost of AI deployment which means that only giant players in the food industry afford it.

Cultural challenges: as with all technical advancements, the deployment of AI is associated with fear. The fear that computers are taking over and the fear that such technologies can be negatively manipulated in future, the fear that power will be left in the hands of few and that humans will loose their jobs to computers. These could lead to companies getting detested from embracing AI [14].

AI technology is still in infancy stage, there is a growing need for specialized skill sets around gathering and analyzing data [1]. As a new technology, many companies are hesitant to invest until it is well known the true value or capability of AI to deliver. AI technology required increased transparency and more involvement of consumers in decision making. This is challenge because food and beverage companies are known to guard their secret recipes fiercely [23]. 
One track minds: the vast majority of AI implementation in use are highly specialised. They carry out one specific task and learn to become better and better at it [24]. It does this by simulating what would happen given every combination of input value and measures the result until the most effective output is achieved.

\section{Conclusion}

Artificial intelligence is making the food industry more efficient and better and promises to yield many more new changes in the near future. Al's role in the food industry is becoming increasingly important due to its ability to reduce waste, predict markets for products, enable round the clock efficient and effective monitoring, augment sanitation, manage cost and increase revenue. Researches are ongoing to profer solutions to the challenges of artificial intelligence and increase its adoption.

\section{Compliance with ethical standards}

\section{Acknowledgments}

The author acknowledges Department of Food Science and Technology, Federal University of Technology Owerri for the supervision of this review.

\section{Disclosure of conflict of interest}

There are no conflicts of interest.

\section{References}

[1] Sebastin, J. Artificial Intelligence: a real opportunity in food industry. Food Quality and Safety. 2018. [cited 2019 April 23]. Available from https://www.foodqualityandsafety.com.

[2] Wang, P. What do you mean by "AI”? Frontiers in Artificial Intelligence and Application, 2008; 171(1): $362-373$.

[3] Kumar C. Artificial intelligence: Definition, types, examples, technologies. [cited 2019 March 20]. Available from http://www.medium.com,

[4] Singh, H. How AI is reshaping the food processing business. Customer think. [cited 2019 April 25]. Available from https://www.customerthink.com.

[5] Kurilyak, S. Artificial Intelligence (AI) in food industry. [cited 2019 April 25]. Available from http://www.produvia.com.

[6] Goyache F, Bahamonde A, Alonso J, López S, Del Coz JJ, Quevedo JR, Ranilla J, Luaces O, Álvarez I, Royo LJ, Díez J. The usefulness of artificial intelligence techniques to assess subjective quality of products in the food industry. Trends in Food Science \& Technology. 2001; 12(10):370-381.

[7] Iqbal J, Khan ZH, Khalid A. Prospects of robotics in food industry. Food Science and Technology. 2017; 37(2): 159165.

[8] Bishop, C. M. Pattern recognition and machine learning. $1^{\text {st }}$ ed. New York, United States: Springer; 2016.

[9] Goldberg Y. A primer on neural network models for natural language processing. Journal of Artificial Intelligence Research. 2016; 57:345-420.

[10] Milan, S., Vaclav, H., and Roger, B. Image processing analysis and machine vision. $3^{\text {rd }}$ ed.. Florence, KY, United States: Cengage Learning; 2008.

[11] Tondu, B. Modelling of the Mckibben artificial muscle: a review. Journal of Intelligent Material Systems and Structures. 2012; 23(3): 225-253.

[12] Wettels, N., Santos, V. J., Johansson, R. S., and Loeb, G. E. Biomimetic tactile sensor array. Advanced Robotics, 2008; 22(8), 829-849.

[13] Thrun, S. Toward robotic cars. Communications of the Association for Computing Machinery (ACM). 2010; 53(4): 99-106.

[14] Hammerkopf. Artificial intelligence in the food industry. [cited 2019 April 25]. Available from http://www.hammerkopf.com. 
[15] Shobhit, S. What are the applications of AI in food industry? [cited 2019 April 25]. Available from https://www.quora.com.

[16] Tan, V. J. Artificial intelligence use cases in Agriculture. [cited 2019 April 25]. Available from https://www.ai.business.com.

[17] Crowbarprotein. Artificial intelligence in the food industry-a complete guide. Available from https://crowbarprotein/.

[18] Walker, J. AI in food processing use cases and applications that matter. [cited 2019 April 25]. Retrieved from https://www.emerj.com.

[19] Linko, S. Expert systems- what can they do for the food industry? Trends in Food Science and Technology. 1998; $9(1): 3-12$.

[20] Sennaar, K. Examples of AI in restaurants and food services. [cited 2019 April 25]. Available from https://www.emerj.com.

[21] Rensi, E. McDonalds says goodbye cashiers, hello kiosks. Forbes. [cited 2019 April 20]. Available from http://www.forbes.com.

[22] Worral, S. Will the rise of the robots implode the world economy? National geographic. [cited 2019 April 25]. Available from https://www.nationalgeographic.com.

[23] Jia, M. Tasting tomorrow: how food and beverage companies use AI. [cited 2019 April 20]. Available from http://www.topbots.com.

[24] Marr B. The biggest challenges facing artificial intelligence (AI) in business and society. [cited 2019 April 20]. Available from https://www.forbes.com/sites/bernardmarr/2017/07/13/the-biggest-challengesfacingartificial-intelligence-ai-in-business-andsociety $/ 3$. 